\title{
K-t-GRAPPA accelerated flow measurements
}

\author{
Bernd A Jung ${ }^{1 *}$, Simon Bauer ${ }^{1}$, Jelena Bock ${ }^{1}$, Michael Markl ${ }^{2}$ \\ From 15th Annual SCMR Scientific Sessions \\ Orlando, FL, USA. 2-5 February 2012
}

\section{Background}

Parallel imaging can reduce scan time with typical reduction factors of $\sim 2$, often not sufficient to reduce scan time to breath hold duration. Spatiotemporal parallel imaging such as k-t-GRAPPA allows for a significantly higher speed-up in data acquisition, but previous studies mostly acquired full $\mathrm{k}$-space data while removing data retrospectively. Here, aortic flow scans were acquired during breath-hold using kt-GRAPPA based reconstruction $[1,2]$ and compared to conventional protocols using GRAPPA and navigator respiration gating.

\section{Methods}

PC imaging in the ascending aorta (venc $1.5 \mathrm{~m} / \mathrm{s}$ ) was performed on a $3 \mathrm{~T}$ system (Trio, Siemens) using a 12channel thorax coil with three different scans in 10 healthy volunteers:

1) Breath-hold using kt-GRAPPA ( $R=5 /$ Rnet $=4.0) ; 2)$ Free-breathing using conventional GRAPPA $(\mathrm{R}=2$ / Rnet=1.7); 3) Free-breathing using kt-GRAPPA ( $R=5 /$ Rnet $=4.0$ ).

Scan parameters were: thickness $8 \mathrm{~mm}$, matrix $256 \times 160(1.3 \times 1.5 \mathrm{~mm})$, temporal resolution $28 \mathrm{~ms}, 6 \mathrm{~mm}$ navigator gating window for free-breathing scans, scan time $12 \mathrm{~s}$ for breath-hold scan $(\mathrm{R}=5)$ and $55 \mathrm{~s}$ for freebreathing scan $(R=2)$ assuming a $50 \%$ navigator efficiency and an RR-interval of $0.9 \mathrm{~s}$. The reconstruction was directly implemented into the Siemens image reconstruction environment. Velocity time courses and peak velocities in the ascending aorta were determined. Further, 4D flow scans were acquired with GRAPPA $(\mathrm{R}=2 / \mathrm{Rnet}=1.6)$, and $\mathrm{kt}$-GRAPPA $(\mathrm{R}=5 / \mathrm{Rnet}=4.4$ and $\mathrm{R}=8 / \mathrm{Rnet}=6.2$ ) in a healthy volunteer using acquisition patterns for $\mathrm{R}=5$ and 8 according to [3]. Streamline visualization and velocity time courses were evaluated and compared.

Dept. of Radiology, Medical Physics, University Medical Center, Freiburg, Germany

Full list of author information is available at the end of the article

\section{Results}

Images in Fig.1a clearly demonstrate improved image quality of the breath-hold scans compared to freebreathing. The mean velocity time courses in Fig.1b show good agreement between kt-GRAPPA and conventional 2D-PC. A slight underestimation of peak velocities by conventional GRAPPA data can be seen whereas both kt-GRAPPA scans demonstrated an excellent agreement and less low pass filtering, corroborated by

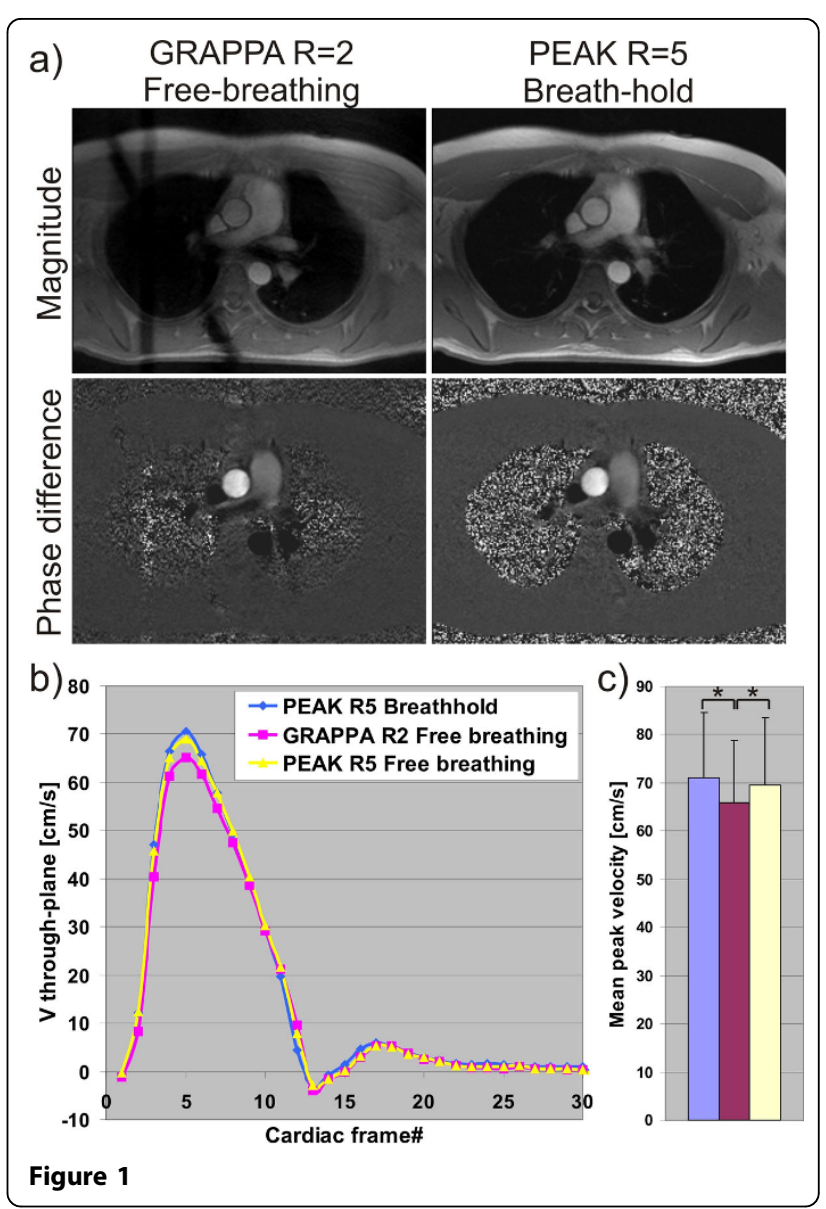




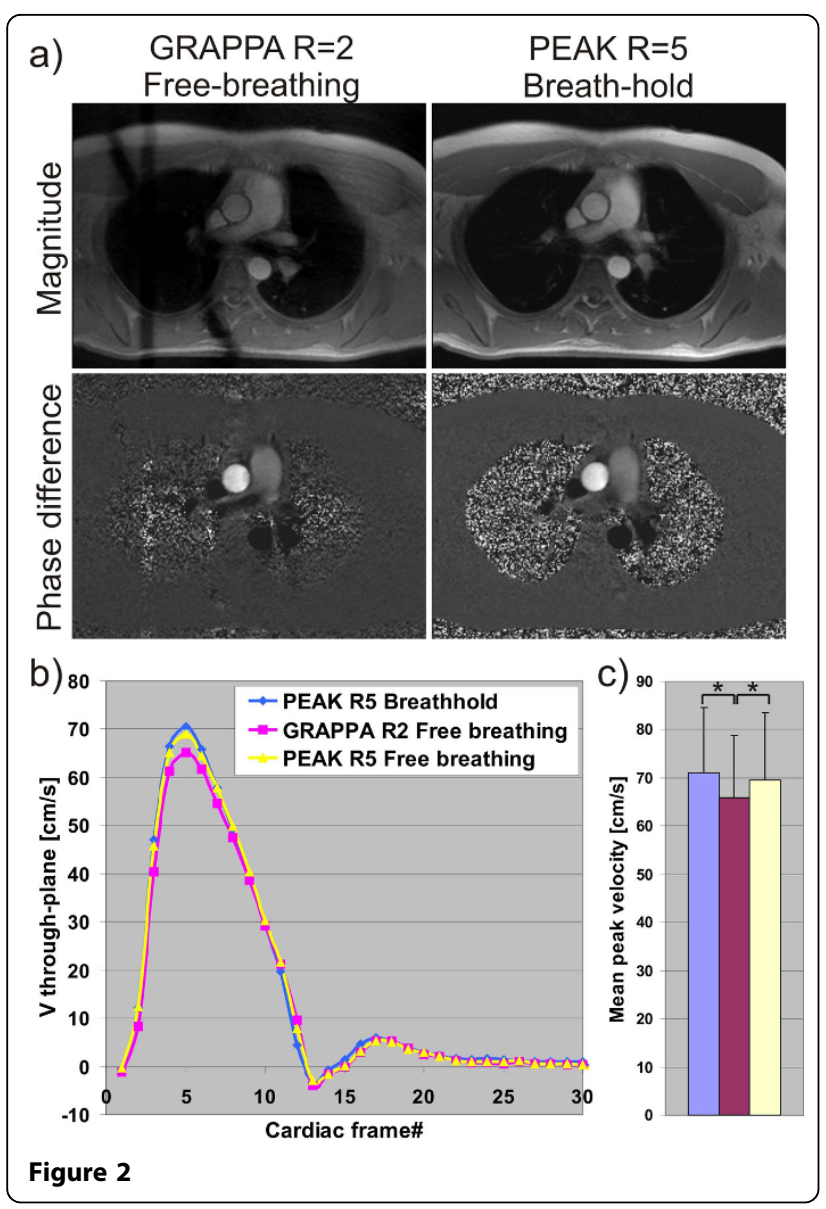

the mean peak velocities in Fig.1c where GRAPPA yielded the lowest peak velocities. Streamline visualization in Fig.2a shows some noise enhancement for ktGRAPPA (b,c) compared to GRAPPA (a) (see angiography), and a good maintenance of the velocity field distribution for $\mathrm{R}=5$ as shown in the flow course in the ascending aorta (d). A slight underestimation of the velocities occurs for $\mathrm{R}=8$.

\section{Conclusions}

The results indicate that the use of accelerations factor of up to $\mathrm{R}=5$ with $\mathrm{kt}$-GRAPPA can provide scan time reductions that allows time-resolved $2 \mathrm{D}$ data acquisition during breath-hold while maintaining a high temporal resolution or $4 \mathrm{D}$ flow scans in $\sim 6-8$ minutes.

\section{Funding}

BMBF 01EV0706

\section{Author details}

${ }^{1}$ Dept. of Radiology, Medical Physics, University Medical Center, Freiburg, Germany. ${ }^{2}$ Dept. of Radiology, Northwestern University, Chicago, IL, USA.

Published: 1 February 2012

\section{References}

1. Jung : MRM. 2008, 60:1169.

2. Huang : MRM. 2005, 54:1172

3. Jung : MRM. 2011, Epub.

doi:10.1186/1532-429X-14-S1-P257

Cite this article as: Jung et al:: K-t-GRAPPA accelerated flow

measurements. Journal of Cardiovascular Magnetic Resonance 201214

(Suppl 1):P257.
Submit your next manuscript to BioMed Central and take full advantage of:

- Convenient online submission

- Thorough peer review

- No space constraints or color figure charges

- Immediate publication on acceptance

- Inclusion in PubMed, CAS, Scopus and Google Scholar

- Research which is freely available for redistribution 